\title{
CONSTITUINTES VOLÁTEIS DE CAFÉS “GOURMET" E MOLE DO CERRADO DO TRIÂNGULO MINEIRO EM FUNÇÃO DA TORRA'
}

\author{
Evandro Afonso do NASCIMENTO ${ }^{2, *}$, Sérgio Antônio Lemos de MORAIS ${ }^{3}$ Rafael Souza ROCHA ${ }^{4}$
}

\begin{abstract}
RESUMO
Neste trabalho foi estudada a variação da composição dos voláteis de dois cafés "gourmet" e de um café mole em função do grau de torrefação. Os cafés provenientes de Araguari, cerrado do Triângulo Mineiro, foram submetidos à torra americana (grãos marrons claros), média (grãos marrons) e forte (grãos pretos) e, em seguida, moídos e submetidos a uma destilação por arraste de vapor em contracorrente com diclorometano. A análise de cromatografia gasosa acoplada à espectrometria de massas (CG-EM) dos constituintes voláteis mostrou que piridina, pirazina e derivados, furfural e derivados são os principais constituintes voláteis dos cafés analisados. Mostrou ainda que não é possivel diferenciar os três tipos de café pelos compostos dominantes (concentração acima de $1 \%$ ) no aroma. Foi observado também que a torra afeta sensivelmente os resultados, sendo que a torra americana, usada normalmente na prova da xícara para classificação sensorial de cafés, produz alguns voláteis de forte impacto no aroma que não aparecem na torra média nem na forte, além de apresentar concentrações dos componentes mais comuns muito diferentes daquelas observadas nas outras duas torras.

Palavras-chave: café torrado; café "gourmet"; voláteis do café.
\end{abstract}

\section{SUMMARY}

VOLATILE CONSTITUENTS OF SOFT AND SPECIAL ARABIAN COFFEES FROM BRAZILIAN “CERRADO” IN FUNCTION OF THE ROASTING GRADE. One soft and two "gourmet" Arabica coffee samples from Brazilian "cerrado" were submitted to three kinds of roasting: american (light brown beans), medium (brown beans), and hard (black beans). The GC/MS analysis showed that the major volatile components (concentration $>1 \%$ ) are pyridine, pyrazine and derivatives, furfural and derivatives. This technique showed that it is not possible to distinguish the three coffee samples by the dominant volatiles. The results showed also that the roasting grade sensibly affects the volatiles composition, furnishing the american roasting a volatile composition quite different from those obtained by medium and hard roasting.

Keywords: coffee; "gourmet" coffee; roasting; coffee volatiles.

\section{1 - INTRODUÇÃO}

O Brasil é o maior produtor mundial de café, mas, devido ao histórico descaso de nossos governantes e produtores, o café colombiano tem mais fama e a capital mundial do café é conhecida como Trieste, cidade situada no norte da Itália e que não cultiva café, mas aí se desenvolvem as melhores pesquisas sobre qualidade deste produto. Recentemente vários grupos de pesquisa no Brasil resolveram resgatar a identidade nacional e aprofundaram as pesquisas nesta área, para evitar constrangimentos que já ocorrem como o de se importar bolsinhas de café da Itália preparadas com cafés de todo o mundo, inclusive com os nossos [6].

Os melhores cafés produzidos no Brasil são os especiais, conhecidos também como cafés "gourmet". Devido ao sabor suave e peculiar, seu consumo no mercado internacional tem aumentado significativamente e o Brasil é o único país que tem condições de sustentar esta demanda no futuro, estimada em 15 milhões de sacas por ano [5]. Entretanto, o consumo de cafés especiais no país ainda é pequeno, devido a falta de divulgação e ao

\footnotetext{
Recebido para publicação em 04/06/2002. Aceito para publicação em 26/12/2002 (000839).

2. Instituto de Química da Universidade Federal de Uberlândia, Av. João Naves de Ávila, 2160, Bairro Santa Mônica, CEP: 38400-902, Uberlândia - MG. E-mail: eanascimento@ufu.br

3. Instituto de Química, Universidade Federal de Uberlândia.

4. Iniciação Científica, Instituto de Química, Universidade Federal de Uberlândia.

* A quem a correspondência deve ser enviada.
}

preço que é de duas a quatro vezes o dos cafés comuns. No entanto, esse mercado vem começando a conquistar espaço internamente, reproduzindo, tardiamente, uma realidade existente no exterior.

É conhecido que a qualidade do café acha-se estritamente relacionada com os diversos constituintes quimicos responsáveis pelo seu aroma e sabor os quais, por sua vez, estão determinados por fatores pré e pós-colheita [2].

Uma boa revisão dos constituintes voláteis presentes no café torrado encontra-se mencionada nas referências $[3,8]$. Recentemente foi verificado que diferentes tipos de irrigação e colheita não puderam ser diferenciados pelos constituintes voláteis e volatilizáveis do café torrado resultante [7].

O presente trabalho procurou identificar os constituintes voláteis presentes em dois cafés do tipo "gourmet" e compará-los com aqueles presentes num café classificado como do tipo "mole". Foram empregados três tipos de torra: a fraca, conhecida também como americana, pela qual os grãos resultantes apresentam coloração marrom clara; a torra média que produz grãos marrons e a torra forte que fornece grãos pretos.

\section{2 - MATERIAIS E MÉTODOS}

Foi usado $1 \mathrm{~kg}$ de amostra de café em grãos selecionados com peneira número 18. A amostra de café arábica "bebida mole" foi obtida na COCACER (Cooperativa dos Cafeicultores do Cerrado de Araguari-MG); as amostras dos cafés especiais ("gourmet") foram fornecidas pelas Fazendas Sacoman e Neoliberto, ambas situadas também 
no municipio de Araguari-MG. Estes cafés foram classificadas entre os dez melhores do ano de 2001 pela Illy Café.

\section{1 - Processo de torrefação}

As amostras, aproximadamente $500 \mathrm{~g}$, foram torradas em um reator elétrico (karmomáquina, modelo 168), sob constante agitação, na COCACER. A torra fraca foi obtida com a temperatura final de $120^{\circ} \mathrm{C}\left( \pm 5^{\circ} \mathrm{C}\right)$ durante 5 minutos. A torra média foi obtida com a temperatura final de $135^{\circ} \mathrm{C}\left( \pm 5^{\circ} \mathrm{C}\right)$ durante 15 minutos. A torra forte foi obtida com a temperatura final de $145^{\circ} \mathrm{C}\left( \pm 5^{\circ} \mathrm{C}\right) \mathrm{du}-$ rante 27 minutos.

\section{2 - Extração por destilação simultânea com diclorometano}

Para este procedimento foi empregado o método de extração simultânea com solventes em escala micro, desenvolvido por GODEFROOT et al [4], trata-se de uma modificação da conhecida aparelhagem de destilação por arraste de vapor de Clevenger.

Três gramas de cada amostra torrada, foram moídas mecanicamente em moinho caseiro e introduzida imediatamente no balão de $50 \mathrm{~mL}$ contendo $20 \mathrm{~mL}$ de água destilada e duas pedrinhas de ebulição e o balão foi aquecido a $115^{\circ} \mathrm{C}$. Ao mesmo tempo, no outro balão de $5 \mathrm{~mL}$ foram colocados $2 \mathrm{~mL}$ de diclorometano (grau cromatográfico) e aqueceu-se a $65^{\circ} \mathrm{C}$. Na coluna de vidro do aparelho foram adicionados $1,5 \mathrm{~mL}$ de diclorometano e $1,5 \mathrm{~mL}$ de água destilada para facilitar a troca de componentes no sistema de fase aquosa e orgânica. Processou-se a extração durante 2 horas e recolheu-se a fase orgânica. Para a análise cromatográfica, a solução foi secada com sulfato de sódio anidro e injetada imediatamente no CG-EM.

\section{3 - Identificação dos componentes voláteis}

Um $\mu \mathrm{L}$ da fase orgânica proveniente da extração simultânea com diclorometano foi injetada num cromatógrafo a gás acoplado a um espectrômetro de massas (SHIMADZU QP 5000). As condições operacionais usadas na cromatografia foram: coluna DB5 de $30 \mathrm{~m}, 0,25 \mu$ de espessura do revestimento interno e $0,25 \mathrm{~mm}$ d.i., programa de temperatura de $60^{\circ} \mathrm{C}$ a $240^{\circ} \mathrm{C}\left(5^{\circ} \mathrm{C} / \mathrm{min}\right)$; temperatura do injetor e do detector de $220^{\circ} \mathrm{C}$ e $240^{\circ} \mathrm{C}$, respectivamente. A espectrometria de massas foi feita com energia de impacto de $70 \mathrm{~V}$ e foram coletados fragmentos de 45 a $650 \mathrm{Da}$. A identificação dos compostos foi baseada nas bibliotecas de espectros de massas (Wiley 140 e 229) e em indices de Kovat (IK) [1]. A curva de calibração usada para obtenção dos indices de Kovat foi: $\mathrm{IK}=23,44 \mathrm{x}$ tempo de retenção (minutos) + 673. O espectro de massas obtido para cada componente e o respectivo indice de Kovat foram comparados com aqueles contidos nas tabelas apresentadas em [1]. A identificação de cada composto se baseou em pelo menos $80 \%$ de indice de similaridade entre os espectros de massas da substância desconhecida e da tabelada e indices de Kovat parecidos $( \pm 30$ pontos de diferença). A quantificação dos componentes resultou da integração da área do respectivo pico e foi fornecida automaticamente pelo aparelho. Cada resultado constituiu a média de três experimentos. O cromatograma foi de íons totais (TIC) de cada componente.

\section{3 - RESULTADOS E DISCUSSÃO}

As Tabelas 1, 2 e 3 apresentam os compostos predominantes nos óleos essenciais obtidos dos cafés "gourmet" das fazendas Neoliberto e Sacoman e do café mole, com as correspondentes quantificações, segundo os diferentes graus de torrefação.

TABELA 1. Voláteis do café Neoliberto - torras fraca, média e forte.

\begin{tabular}{|c|c|c|c|c|}
\hline \multirow[b]{2}{*}{ COMPOSTO } & \multirow{2}{*}{$\begin{array}{l}\text { TEMPO DE } \\
\text { RETENÇÃO } \\
\text { (min) }\end{array}$} & \multicolumn{3}{|c|}{$\begin{array}{c}\text { INTENSIDADE DO PICO } \\
(\%) / \text { TORRA }\end{array}$} \\
\hline & & FRACA & MÉDIA & FORTE \\
\hline Alil propil éter & 2,419 & 2,88 & & \\
\hline 2-metilbutanal & 2,537 & 7,60 & 0,85 & \\
\hline Pentan-2,3-diona & 2,873 & 7,71 & & \\
\hline n.i. & 3,127 & & 0,99 & \\
\hline Pirazina & 3,590 & & 1,98 & 1,29 \\
\hline 1-metil-1-pirrol & 3,764 & 2,23 & & 0,76 \\
\hline Piridina & 3,838 & 14,10 & 26,59 & 69,29 \\
\hline Pirrol & 3,970 & & & 1,13 \\
\hline Tolueno & 4,350 & 1,86 & & \\
\hline 2-metil-tetraidrofuran-3-ona & 5,191 & 1,40 & 2,31 & \\
\hline 2-metilpirazina & 5,706 & 4,14 & 7,29 & 3,78 \\
\hline n.i. & 5,838 & & 2,56 & 0,92 \\
\hline Furfural & 5,948 & 7,81 & 3,87 & 0,83 \\
\hline Álcool furfurílico & 6,530 & 1,40 & 30,25 & 9,42 \\
\hline Etilbenzeno & 7,036 & 5,43 & & \\
\hline Xileno & 7,294 & 23,25 & 2,34 & 1,05 \\
\hline Estireno & 8,062 & 2,34 & & \\
\hline Xileno (isômero) & 8,169 & 8,11 & & \\
\hline 2,6-dimetilpirazina & 8,693 & 2,13 & 4,42 & 2,52 \\
\hline Etil-pirazina & 8,872 & 1,26 & 1,26 & \\
\hline n.i. & 10,577 & & 1,12 & \\
\hline 5-metilfurfural & 10,815 & 3,95 & 6,68 & 1,10 \\
\hline Fenol & 11,454 & & & 2,48 \\
\hline Acetato de furfurila & 12,128 & 2,00 & 2,54 & 2,08 \\
\hline 2-etil-3-metilpirazina & 12,580 & & 1,28 & 1,03 \\
\hline 1-metil-2-pirrol carboxaldeído & 12,790 & & 1,44 & 0,79 \\
\hline Guaiacol & 16,830 & & & 1,54 \\
\hline 1-(2-furfuril)-pirrol & 21,420 & & 1,04 & \\
\hline 3-(2-furanil)-penta-3-en-2-ona & 27,849 & & 1,19 & \\
\hline
\end{tabular}

n. i.: não identificado

Os espaços em branco nestas tabelas significam que o composto não foi detectado ou que foi observado em concentração relativamente baixa. As substâncias principais são relativamente poucas tendo em vista que já se identificaram centenas de constituintes em cafês torrados [3, 7]. A grande maioria, no entanto, está em baixa concentração, o que faz com que este número se restrinja enormemente. A comparação das três tabelas revela várias regularidades:

- $\quad$ piridina, pirazina e derivados, 2-metil-tetraidrofuran-3ona, furfural e derivados estão quase sempre presentes;

- os compostos mais leves (situados acima da pirazina) perdem importância ao se passar da torra fraca para a média, ao passo que os mais pesados (situados abaixo do acetato de furfurila) ganham importância;

- tolueno, etilbenzeno, xilenos e estireno têm um comportamento semelhante ao mencionado acima;

- piridina e álcool furfurílico são muito sensiveis à temperatura da torra, principalmente ao se passar da torra americana para a média; 
TABELA 2. Voláteis do café Sacoman - torras fraca, média e forte.

\begin{tabular}{|c|c|c|c|c|}
\hline \multirow[b]{2}{*}{ COMPOSTO } & \multirow{2}{*}{$\begin{array}{l}\text { TEMPO DE } \\
\text { RET ENÇÃO } \\
\text { (min) }\end{array}$} & \multicolumn{3}{|c|}{$\begin{array}{l}\text { INTENSIDADE DO PICO (\%) } \\
\text { TORRA }\end{array}$} \\
\hline & & FRACA & MÉDIA & FORTE \\
\hline Alil propil éter & 2,419 & 1,85 & & \\
\hline 2-metilbutanal & 2,537 & 4,99 & & \\
\hline Benzeno & 2,590 & & & 1,63 \\
\hline Pentan-2,3-diona & 2,873 & 4,64 & & \\
\hline Pirazina & 3,590 & 1,60 & 1,47 & \\
\hline Piridina & 3,838 & 15,72 & 71,87 & 14,67 \\
\hline Pirrol & 3,970 & 1,66 & 0,93 & \\
\hline 2-metil-tetraidrofuran-3-ona & 5,191 & 2,71 & & 1,94 \\
\hline 2-metilpirazina & 5,706 & 9,92 & 3,57 & 5,21 \\
\hline n.i. & 5,838 & 1,44 & 0,91 & 2,05 \\
\hline Anidrido acético & 5,891 & & & 1,89 \\
\hline Furfural & 5,948 & 16,06 & & 3,84 \\
\hline Álcool furfurílico & 6,530 & 5,68 & 8,18 & 34,23 \\
\hline Etilbenzeno & 7,036 & 1,86 & & \\
\hline Xileno & 7,294 & 8,33 & 2,28 & 1,12 \\
\hline Xileno (isômero) & 8,169 & 2,62 & & \\
\hline $\begin{array}{l}\text { 2-etil-5-metilfurano + 2,6- } \\
\text { dimetilpirazina }\end{array}$ & 8,693 & 6,22 & 2,91 & 5,34 \\
\hline Etil-pirazina & 8,872 & 1,90 & & 1,02 \\
\hline n.i. & 10,577 & & & 1,44 \\
\hline n.i. & 10,715 & & & 0,98 \\
\hline 5-metilfurfural & 10,815 & 8,45 & 0,92 & 7,67 \\
\hline Fenol & 11,454 & & 2,85 & 3,03 \\
\hline Acetato de furfurila & 12,128 & 1,38 & 1,57 & 1,99 \\
\hline 2-etil-5-metilpirazina & 12,425 & 1,10 & & \\
\hline 2-etil-3-metilpirazina & 12,575 & 1,88 & 1,21 & 1,37 \\
\hline $\begin{array}{l}\text { 1-metil-2- } \\
\text { pirrolcarboxaldeído }\end{array}$ & 12,779 & & & 1,31 \\
\hline 2,3,5-trimetilpirazina & 13,329 & & & 0,99 \\
\hline Acetilpirrol & 15,238 & & & 1,17 \\
\hline Guaiacol & 16,806 & & 1,41 & 1,45 \\
\hline 1-(2-furfuril)-pirrol & 21,419 & & & 1,35 \\
\hline 2,5-dimetoxitolueno & 26,185 & & & 1,05 \\
\hline Difurfuriléter & 27,100 & & & 0,96 \\
\hline $\begin{array}{l}\text { 3-(2-furanil)-penta-3-3n-2- } \\
\text { ona }\end{array}$ & 27,851 & & & 2,33 \\
\hline
\end{tabular}

TABELA 3. Voláteis do café mole - torras fraca, média e forte.

\begin{tabular}{|c|c|c|c|c|}
\hline \multirow[b]{2}{*}{ COMPOSTO } & \multirow{2}{*}{$\begin{array}{l}\text { TEMPO DE } \\
\text { RETENÇÃO } \\
\text { (min) }\end{array}$} & \multicolumn{3}{|c|}{$\begin{array}{c}\text { INTENSIDADE DO PICO (\%) / } \\
\text { TORRA }\end{array}$} \\
\hline & & FRACA & $\begin{array}{l}\text { TORRA } \\
\text { MÉDIA }\end{array}$ & FORTE \\
\hline 2-metilbutanal & 2,537 & 6,03 & 1,23 & 1,33 \\
\hline Pentan-2,3-diona & 2,873 & 5,74 & & \\
\hline n.i. & 3,135 & & 1,09 & \\
\hline Pirazina & 3,590 & 2,96 & 2,30 & 1,25 \\
\hline Piridina & 3,838 & & 34,75 & 52,51 \\
\hline Pirrol & 3,970 & & & 1,11 \\
\hline Tolueno & 4,380 & 2,49 & & \\
\hline n.i. & 5,029 & 2,38 & & \\
\hline 2-metil-tetraidrofuran-3-ona & 5,191 & 2,76 & 2,40 & 1,49 \\
\hline 2-metilpirazina & 5,706 & 3,78 & 6,03 & 5,72 \\
\hline Ácido isovalerico & 5,838 & & 2,08 & \\
\hline Furfural & 5,948 & 14,39 & 5,27 & 1,77 \\
\hline Álcool furfur ílico & 6,530 & 4,05 & 22,89 & 17,66 \\
\hline Etilbenzeno & 7,036 & 6,40 & & \\
\hline Xileno & 7,294 & 25,79 & 3,51 & 2,38 \\
\hline Estireno & 8,086 & 3,21 & & \\
\hline Xileno (isômero) & 8,169 & 10,69 & 1,35 & 1,00 \\
\hline $\begin{array}{l}\text { 2-etil-5-metilfurano + 2,6- } \\
\text { dimetilpirazina }\end{array}$ & 8,693 & 3,07 & 3,94 & 3,12 \\
\hline 5-metilfurfural & 10,815 & 6,25 & 7,36 & 2,59 \\
\hline Fenol & 11,454 & & & 1,37 \\
\hline Acetato de furfurila & 12,128 & & 2,25 & 3,06 \\
\hline 2-etil-3-metilpirazina & 12,575 & & 1,11 & 1,30 \\
\hline $\begin{array}{l}\text { 1-metil-2- } \\
\text { pirrolcarboxaldeído }\end{array}$ & 12,779 & & 1,38 & 1,12 \\
\hline Guaiacol & 16,806 & & & 1,24 \\
\hline 1-(2-furfuril)-pirrol & 21,419 & & 1,08 & \\
\hline
\end{tabular}

- furfural perde importância ao se aumentar a temperatura de torra;

- $\quad$ pirazina e derivados são pouco sensiveis à temperatura de torra, o mesmo é válido para 5-metilfurfural;

- nas torras média e forte os dois constituintes destacadamente dominantes são a piridina e o álcool furfurílico;
- os constituintes e suas porcentagens observados na torra americana são significativamente diferentes daqueles observados nas torras média e forte. Considerando-se que, atualmente, a prova de degustação (prova da xícara) é feita com grãos torrados na torra americana, esta constatação deixa dúvidas quanto à eficácia desta metodologia de classificação de cafés. Isto sem considerar que ela já é intrinsecamente subjetiva, pois depende dos sentidos do degustador na hora da análise.

\section{4 - CONCLUSÕES}

A partir dos constituintes voláteis dominantes no aroma, não foi possivel diferenciar os três tipos de cafés estudados uma vez que não se observaram diferenças fundamentais entre os dois cafés "gourmet" nem entre eles e o café mole. O tipo de torra exerce significativa influência na composição dos compostos voláteis. A torra americana fornece resultados diferentes das torras média e forte. Este resultado lança dúvidas quanto à classificação de cafés via prova da xícara, a qual se baseia neste tipo de torra.

\section{5 - REFERÊNCIAS}

[1] ADAMS, R. P. Identification of essential oil components by GC/MS. Allured Publishing Corp., Carol Stream, USA, 1995, 469 p.

[2] AMSTALDEN, L. C.; LEITE, F.; MENEZES, H. C. Identificação e quantificação de voláteis de café através da cromatografia gasosa de alta resolução / espectrometria de massas empregando um amostrador automático de "headspace". Ciênc. Tecnol. Aliment., v. 21, no 1, p. $123-128,2001$.

[3] DE MARIA, C. A. B.; MOREIRA, R. F. A.; TRUGO, L. C. Componentes voláteis do café torrado. Parte I: compostos heterocíclicos. Química Nova, v. 22, no 2, p.209 - 217, 1999.

[4] GODEFROOT, M.; SANDRA, P.; VERZELE, M. New method for quantitative essential oil analysis. Journal of Chromatography, v.203, p.325 - 335, 1981.

[5] MOREIRA, A. C. O mestre dos sabores. Panorama Atual, v. 19, no 9 , p. $74,2000$.

[6] NASCIMENTO, E. A.; AQUINO, F. J. T. Anais do II Simpósio de Pesquisa dos Cafés do Brasil, Vitória - ES, 24 a 27 de setembro, 2001.

[7] NASCIMENTO, E. A.; MORAIS, S. A.; CHANG, R.; AQUINO, F. J. T. Estudo dos constituintes voláteis e volatilizáveis do café torrado do cerrado e efeito da colheita e irrigação em sua composição. Revista Ceres, v. 49, no 283, p. 295-307, 2002.

[8] TRUGO, L. C.; DE MARIA, C. A. B.; MOREIRA, R. F. A. Componentes voláteis do café torrado. Parte II. Compostos alifáticos, alicíclicos e aromáticos. Química Nova, v. 23, no 2, p. 195 - 203, 2000 .

\section{6 - AGRADECIMENTOS}

À Cooperativa de Cafeicultores do Cerrado (COCACER) e fazendas Sacoman e Neoliberto pelas amostras de café analisadas. 